\title{
40 años de televisión infantil en España (1970-2010)
}

\section{0 years of children's television in Spain (1970-2010)}

\section{Beatriz Feijoo Fernández. Universidad de Vigo (beafeijoo@uvigo.es)}

Aurora García González. Universidad de Vigo (auroragg@uvigo.es)

Recibido el: 09/11/15 - Aceptado el: 03/03/16

\section{Resumen:}

Dado que la televisión, desde su nacimiento, siempre ha sido un medio cercano a los niños, surgió el interés por analizar el grado de atención que la TV dedicó a su audiencia infantil en los principales 40 años de su historia en España. Así, se estudió la evolución del contenido infantil en las parrillas de las cadenas generalistas en abierto desde 1970 a 2010 empleando para ello la técnica del análisis de contenido. Entre las conclusiones destaca el trato poco favorable que las cadenas brindaron a la programación infantil, traducido en un descenso en las horas de emisión hasta su completa desaparición de la oferta generalista, además de reposiciones de contenidos y modificaciones constantes que condicionaban su estabilidad en la parrilla televisiva nacional.

\section{Palabras clave:}

Televisión infantil; niños; estrategia de programación; parrillas TV; cadenas generalistas.

\section{Abstract:}

Given the interest that the television has displayed to children since its origin, it would be profitable analysing the attention that TV dedicated to child audience in its main 40-year history in Spain. Thus, the evolution of children's content was studied in Tv guides of the general-interest channels from 1970 to 2010, using the content analysis. Regarding with the main findings highlight an unfavorable treatment provided to children's programs from general-interest channels, expounded in a decrease of broadcasting hours until the complete disappearance of this content in the general offer, besides replacements and constant changes that conditioned its stability on national television programming.

\section{Keywords:}

Children's TV; children; programming strategy; child audience; tv guide; general-interest channels. 


\section{Introducción}

"La oferta de todas las televisiones nacionales generalistas incluye programación infantil desde sus primeras emisiones regulares” (Vázquez Barrio, 2011: 38). Desde finales de los años setenta, La 1 ya emitía contenidos para los niños todas las tardes de la semana tras finalizar el horario escolar. Hasta la aparición de las cadenas privadas, el canal público fue incrementando el tiempo de emisión dedicado a los más pequeños en la franja de la mañana y de sobremesa, también en los fines de semana.

Con la competencia de las televisiones privadas, como Telecinco y Antena 3 que no descuidaron a los niños en su oferta televisiva inicial, las dos cadenas estatales redujeron su nivel de atención hacia este público; no obstante, La 2 se convirtió en el principal contenedor de contenidos infantiles por parte del ente público. La implantación de la televisión digital aumentó la variedad y la cantidad de formatos televisivos lo que fragmentó las audiencias y provocó que las cadenas generalistas abandonasen "el proyecto de crear una programación equilibrada para todos los segmentos sociales para privilegiar contenidos susceptibles de interesar al mayor número de espectadores” (Vázquez Barrio, 2011: 39), una estrategia de programación desfavorable para el niño.

En este estudio se entiende por contenido infantil no sólo aquel concebido y diseñado para un público infantil sino todos aquellos programas y series (sobre todo) "para niños" y "con niños" (que no infantiles) emitidos en las franjas horarias más afines a este perfil de espectador. Desde mediados de la década de los 70, la tarde (de 17.00 a 20.30) ha sido el momento del día más idóneo para programar para la infancia, al que se unió la franja despertador (de 7.30 a 9.00) y las mañanas de los fines de semana (Vázquez Barrio, 2011). La primera hora de la mañana y la segunda mitad de la tarde de lunes a viernes, sábados y domingos concentraron durante más de 20 años la mayoría de la producción infantil emitida en España. No obstante, nuevas estrategias de las cadenas generalistas de programar para el máximo número de espectadores y la fragmentación de las audiencias que supuso la TDT, han ido provocando el debilitamiento y hasta la desaparición de las franjas horarias más afines a los niños, un ejemplo es la tarde de los días laborables en las que se sacrificaron los contenidos infantiles por otros programas destinados al público adulto (amas de casa, personas de más edad, población activa que vuelve del trabajo...).

Aun así, se incluye dentro de esta clasificación de programas infantiles todo aquel producto audiovisual que, aunque no fuese concebido para el público infantil en concreto, fuese emitido en las franjas horarias anteriormente descritas. Series extranjeras, como El Equipo A o McGyver, o dibujos animados como Padre de Familia o American Dad, son ejemplos que fueron retransmitidos como contenido de programas contenedores (Megatrix, Desesperado Club Social) o a través de cadenas temáticas infantiles, a pesar de no ser producciones para una audiencia infantil. 


\section{Objetivos y método}

El objetivo principal de esta investigación es describir la evolución y cambios que vivió la programación infantil en los primeros 40 años de televisión en España.

Concretamente, se busca diferenciar y comparar las etapas por las que fueron pasando los programas destinados a los niños en las parrillas televisivas, prestando especial atención a las franjas horarias de emisión, al formato empleado, a su duración, a su tiempo en antena o a su producción.

Se trata, en definitiva, de definir el tratamiento que las cadenas generalistas brindaron a la programación infantil para finalmente comprobar la evolución de sus estrategias de programación asociadas a este tipo de contenido.

Para ello se recurrió a la observación documental y a su técnica de "mayor prestigio científico" (Sierra Bravo, 2001: 287), el análisis de contenido que Berelson definió como una técnica de investigación para la descripción objetiva, sistemática y cuantitativa del contenido manifiesto de las comunicaciones, con el fin de interpretarlas. Para Sierra Bravo (2001: 288) "se trata de observar y reconocer el significado de los elementos que forman los documentos y clasificarlos adecuadamente para su análisis y explicación posterior".

La fuente escogida para el muestreo fueron las parrillas de programación publicadas en el Diario ABC dadas las facilidades de consulta que este periódico brinda a los investigadores al disponer de una hemeroteca en línea (http://hemeroteca.abc.es/) y de acceso totalmente gratuito en la que se pueden consultar y descargar cada una de las páginas de los ejemplares publicados desde 1903.

La muestra también está acotada temporalmente. Este análisis examina la oferta televisiva nacional en abierto de 1970 a 2010: el estudio se inició en la década de los 70 porque supuso el primer momento en la historia de España en el que la televisión se comenzaba a postular como la principal forma de entretenimiento para la población, con una parrilla de emisión estable y un modelo de financiación basado en los ingresos por publicidad. Esta investigación se delimita a 2010 ya que fue el año en el que la TDT se implantó definitivamente en España, lo que posibilitó la expansión de las cadenas temáticas en abierto, muchas de ellas únicas contenedoras de la programación infantil.

Se generó una muestra aleatoria inicial de 12 periódicos por año analizado, un ejemplar por cada mes alternando los días hábiles de la semana. No obstante, dada la vinculación de los contenidos infantiles con la programación en fin de semana, cada tres meses de cada año se analizaron a mayores las parrillas de un sábado y un domingo escogidos al azar procurando además alternar los meses de revisión (si en un año se examinaban los ejemplares de un fin de semana de enero-abril-juliooctubre, para el siguiente año tocarían los de febrero-mayo-agosto-noviembre...). Como consecuencia, finalmente se revisaron 20 periódicos por año, lo que se corresponde con un total de 800 ejemplares del Diario ABC revisados desde 1970 a 2010. 
Para ello, se elaboró una ficha de análisis para estudiar cada programa infantil detectado, con el fin de obtener datos homogéneos para ser comparados y tratados y extraer conclusiones. Las variables de estudio que se tuvieron en cuenta para analizar cada contenido han sido:

1. Nombre del programa.

2. Cadena/s por la que se emite/emitió el programa.

3. Año en que el programa aparece en antena.

4. Ciclo de vida del programa: Menos de 1 año/ De 1 a 2 años/ De 2 a 4 años/ De 4 a 6 años/ De 6 a 8 años/ De 8 a 10 años/ + de 10 años).

5. Tiempo medio en antena por emisión: Menos de 6 meses/ De 6 meses a 1 año/ De 1 a 2 años/ De 2 a 3 años/ De 3 a 4 años/ De 4 a 5 años/ + de 5 años)

6. Franja/s de emisión del programa: Despertador (7.30-9.00) / Matinal (9.00-13.00)/ Access sobremesa (13.00-15.00)/ Sobremesa (15.00-18.00)/ Tarde (18.00-20.00)/ Access prime time (20.00-21.00)/ Prime time (21.00-24.00)/ Latenight (00.00-2.30)/ Madrugada (2.30-7.30).

7. Periocidad de emisión del programa: Diario, de lunes a viernes/Diario, de lunes a domingo/ Fin de semana/ Semanal/ Especial verano/Navidad.

8. Duración del programa: (en minutos).

9. Formato del programa: Programa contenedor/ Dibujos animados/ Serie nacional/ Serie extranjera/ Telenovela/ Película/ Concurso/ Deporte/ Programa musical/ Documental/ Magazinel Talk-show/ Otros.

10. Producción del programa: Nacional o extranjera.

11. ¿El programa ha tenido una emisión continua? En caso negativo, № de veces que se emitió el programa.

12. ¿El programa se ha emitido sólo por una cadena?

13. ¿El programa ha sufrido modificaciones con frecuencia en la parrilla de televisión?

3. La televisión y su audiencia infantil

Muchos estudios reflejan el nivel de penetración que tienen estas pantallas en relación a la edad del usuario. Ahora bien, también sería interesante conocer el consumo infantil de televisión en el contexto global de penetración del medio, con el objetivo de visualizar su presencia en el conjunto total de usuarios y qué grado de importancia tienen los pequeños como espectadores.

82 | n² 22, pp. 79-104 | doxa.comunicación 
Los espectadores entre 4 y 12 años y 13 y 24 años son los que menos minutos ven la televisión al día, con 147 y 133 minutos diarios respectivamente, un consumo muy por debajo de la media situada en 246 minutos/día (Kantar Media, diciembre 2015). Como se puede observar en el cuadro, el tiempo de visionado crece conforme más edad tiene la audiencia, alcanzando su máximo entre los mayores de 65 años, que además de duplicar el consumo hecho por los más jóvenes, fueron el único target de edad que aumentó el número de minutos dedicado a televisión con respecto a 2014.

Cuadro 1: Evolución del consumo de TV por franjas de edad (minutos diarios)

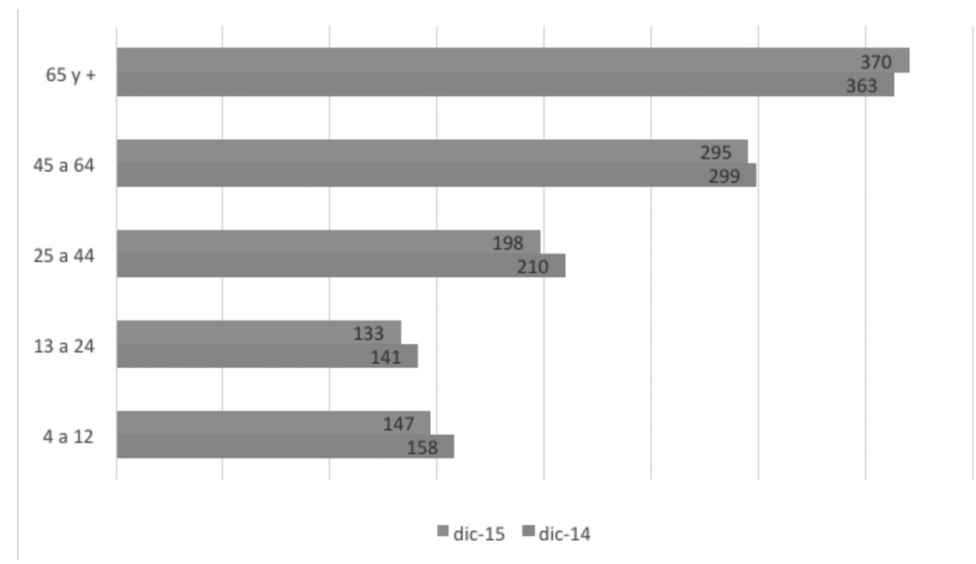

Fuente: Kantar Media 2015

La población de 4 a 12 años es mayoría frente al televisor a primera hora de la mañana, desde las 7.30 hasta las 11.30. Durante el resto del día, el público principal pasa a ser el de 65 o más años, a excepción de la medianoche y madrugada, franjas horarias en las que hubo más espectadores de 45 a 64 años.

Al observar el cuadro 2, se puede comprobar que los niños de 4 a 12 años se convierten en los principales espectadores de la franja despertador. No obstante, no se corresponde con el momento del día de mayor consumo infantil ya que el punto máximo se sitúa entre las 21.30 y 22.30 , horario de prime time. 
Cuadro 2: Franjas horarias de consumo de televisión distribuidas por grupos de edad

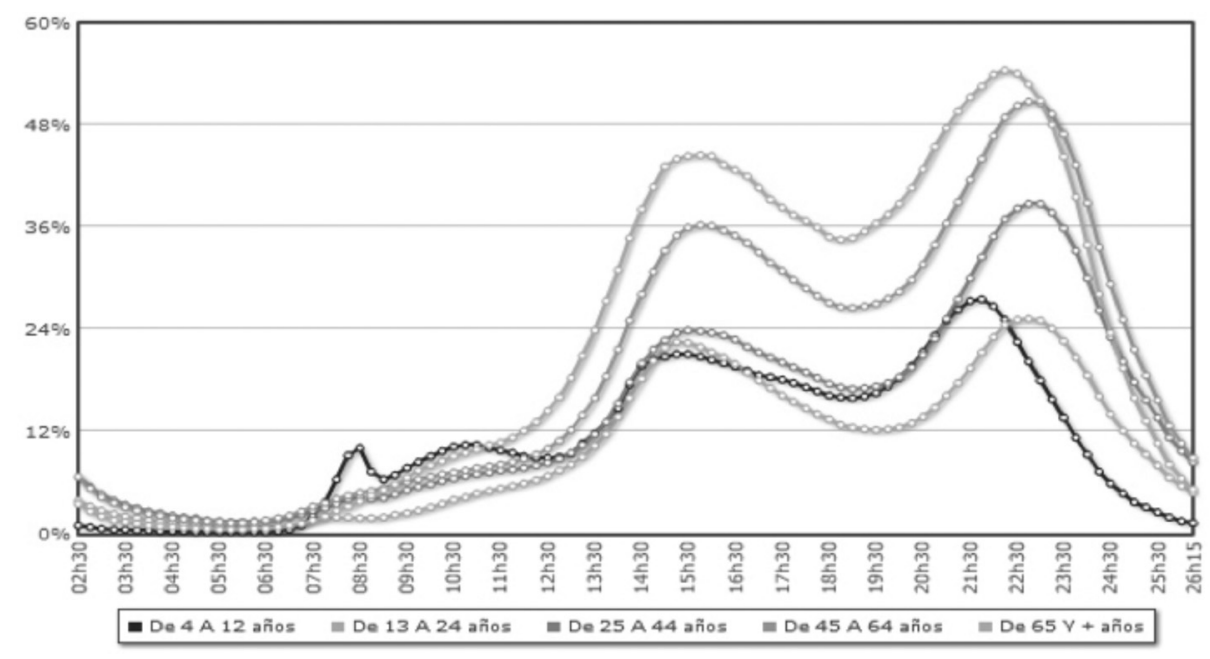

Fuente: Kantar Media 2012

Parece entonces coherente que los niños no sean un objetivo prioritario para las cadenas (Ortiz, Ruiz y Díaz, 2013: 137). Las autonómicas y TVE, por su perfil público, supondrían una excepción a lo anteriormente dicho: mientras que las primeras han tomado ciertas medidas (sobre todo a través de sus libros de estilo) para promocionar entre los profesionales los temas de infancia, TVE ha ido más allá de su función informativa invirtiendo en investigación. Así, autores como Ortiz, Ruiz y Díaz han encontrado en bases documentales de RTVE informes sobre televisión y niños de 1965 (2013: 139).

Por su parte las televisiones privadas se han limitado en su mayoría a suscribir el Código de Autorregulación sobre Contenidos Televisivos e Infancia, vigente desde 2004. Este código establece una serie de principios encaminados a mejorar la protección de la infancia mediante la definición de franjas horarias de protección infantil y de un sistema de clasificación y señalización de contenidos en función de la edad con el objetivo de ayudar a los padres a seleccionar los programas más idóneos para sus hijos.

A tenor de los resultados del seguimiento sobre el grado de complimiento por estas cadenas, da la impresión de que los operadores privados de televisión suscribieron esos acuerdos más por la presión social y mediática que por una manifiesta preocupación por el tema (Ortiz, Ruiz y Díaz, 2013: 142).

Melgarejo y Rodríguez Rosell también reconocen que el carácter voluntario de este código deriva en una sensación de impunidad de las cadenas cuyas estrategias de programación y de contenido distan mucho de lo establecido por la 
autorregulación. "Ni se respetan las franjas horarias de protección al menor, ni se preserva la integridad de los mismos ante las emisiones de contenidos no aptos y mucho menos se sanciona a las televisiones por las malas prácticas realizadas" (2013: 139).

Por otra parte, el proceso de convergencia mediática no contribuye a mejorar el cumplimiento de este Código de Autorregulación. El consumo de contenidos televisivos por internet complica más el control del acceso.

Además de firmar el Código de Autorregulación sobre Contenidos Televisivos e Infancia, la televisión privada poco más se ha interesado por la audiencia infantil. En cadenas de temática infantil en abierto destaca alguna campaña de Responsabilidad Social Corporativa de las corporaciones a favor del menor, como 12 meses, 12 causas de Telecinco, o El Estirón de Antena 3, que promociona hábitos saludables para combatir la obesidad infantil. En relación con las televisiones privadas, "se observa una prevalencia de los aspectos comerciales frente a la infancia" (Ortiz, Ruiz y Díaz, 2013: 143).

Más allá del negocio televisivo están las iniciativas y estudios impulsados por diversas asociaciones, como la Unión de Televisiones Comerciales Asociadas (Uteca) o la Asociación de Consumidores de Usuarios de los Medios (ICmedia) que en 2010 elaboró una guía de los programas más adecuados para cada franja escolar.

Antes de la llegada de la TDT, "los canales generalistas olvidaron casi por completo la emisión de contenidos destinados a los menores" (Melgarejo y Rodríguez Rosell, 2013: 142) lo que convirtió a los niños en una audiencia desatendida que pasó a ser objetivo de las cadenas de pago. Siguiendo datos ofrecidos por estas investigadoras extraídos de un estudio del Consejo Especialista en Canales Temáticos en 2010, el público infantil es el primer target que más consume cadenas de pago (2013: 136).

La TDT impulsó la aparición en abierto de canales de temática infantil, como Clan TV, Boing o Disney Channel, pensados para niños y en los que prima el entretenimiento. No obstante hay que tener en cuenta que más horas de programación infantil no es sinónimo de calidad (Ortiz, Ruiz y Díaz, 2013: 143).

Aunque en números absolutos la audiencia infantil (4-12 años) tenga menos peso que otros grupos de edad, el grado de afinidad entre las cadenas temáticas y los niños quedó patente al ser el tipo de televisión que más consumen (por delante de las generalistas) y al mismo tiempo corresponderse con el perfil de espectador en que las temáticas gratuitas lograron mayor penetración, sin olvidar la presencia destacada de los contenidos infantiles entre las emisiones más vistas de la TDT (en 2010).

\subsection{El niño, un espectador con rol de consumidor}

El niño como espectador es de interés para los medios por su rol de consumidor marcado por su instantaneidad, por su aprecio por las marcas y por su estilo imitativo (Salgado Carrión, 2006). 
El niño en su papel de consumidor se define en función de dos factores, la edad y su entorno de influencia (padres y peer groups). McNeal (1992) propuso cinco etapas en el desarrollo del comportamiento de niño como consumidor; el menor de 12 años objeto de esta investigación ya ha superado las fases de observación, solicitud, selección y compra con ayuda y se encuentra en el último escalón; es un niño que ya puede adquirir bienes de forma independiente, puede hacer compras por sí solo y “además comienza a manejar estrategias de persuasión para convencer a los padres” (Ruiz, s.f.) para realizar compras que implican un cierto desembolso. Para Roedder (1999), que propone tres estados en la consolidación del pequeño como consumidor, el estudiante de sexto de primaria se encontraría en su último estadio definido, el reflexivo, tras completar el perceptivo y el analítico.

Comprenden los significados relacionados con el consumo, tienen mejores formas de validar una percepción y son capaces de incorporar perspectivas ajenas a la suya a la hora de tomar decisiones. Aflora la necesidad de construir su propia identidad, instrumentalizando para ello el consumo. El consumo se supedita a las afinidades identitarias y afectivas con aquellos productos que saben proyectar su entidad y reinterpretarla de forma adecuada (Pérez Alonso-Geta y Sánchez Peris, 2010: 5).

El menor es de gran interés para el mercado y para el sector publicitario porque al mismo tiempo representa varios tipos de mercado: primario, de futuro y de influencia (McNeal, 1992). El primer mercado hace referencia a todos los bienes y servicios que el niño adquiere de forma directa con su propio dinero, más bien cantidades discretas. Suelen adquirir productos como chucherías, cromos, tebeos, helados... "que los convierten, a pesar de su corta edad, en clientes reales para determinados sectores" (Ruiz, s.f.). El mercado de futuro es aquél para el que "adquieren determinados conocimientos y actitudes sobre marcas y productos que todavía no están a su alcance" (Bringué, 2001:1) para convertirse en los compradores de mañana; por último, el mercado de influencia, el más importante para los anunciantes, ya que se refiere no tanto a su nivel adquisitivo sino al poder de influencia que los pequeños ejercen en las decisiones de compra de sus padres. Esta capacidad de influencia se acrecienta con la edad del niño y puede abarcar tanto pequeñas compras como otras que impliquen un mayor gasto.

El peso de las opiniones de los pequeños en las compras familiares es cada vez mayor (Salgado Carrión, 2006; Tur Viñes y Ramos, 2008) motivado por diversos cambios sociológicos sucedidos a lo largo de estos últimos años, entre los que destaca una mayor disponibilidad de ingresos por niño debido a la incorporación de la mujer al mundo laboral y a la tendencia de tener menos descendencia; el incremento de padres separados, más proclives a conceder caprichos; el papel de los abuelos, más presentes en la educación de sus nietos (consecuencia de que ambos padres trabajen) y con mayor disposición de renta o la democratización de la familia que concede más voz al menor. 


\section{La programación infantil en televisión en España (1970-2010)}

Siguiendo la propuesta de Vázquez Barrio (2011) en este trabajo se abordó el estudio de la programación infantil televisiva en etapas definidas en función de los principales hitos que marcaron la historia del medio en España: la aparición de las televisiones privadas y la adaptación a la TDT. Se fijaron tres períodos de análisis avalados por la comunidad científica, pero delimitados de una forma particular para facilitar la búsqueda y el análisis de los programas infantiles.

Los 40 años de programación infantil estudiados empiezan con una primera etapa de 1970 a 1988, último año de monopolio de TVE; la segunda época se delimita entre 1989 y 2008, caracterizada por el nacimiento y consolidación de las televisiones comerciales y por último un tercer período, desde 2009 a 2010. Esta tercera etapa coincide con el proceso del apagón analógico ${ }^{1}$ gracias al cual la TDT se fue expandiendo por todos los hogares españoles.

En la primera etapa (1975-1988) casi el 80\% de los contenidos infantiles (262 programas ${ }^{2}$ ) se emitieron por La 1. En ese momento, La 2 complementaba la oferta de este tipo de programación de la primera cadena.

Cuadro 3: Principales características de la programación infantil según períodos

\begin{tabular}{|c|c|c|c|c|}
\hline & \multirow{2}{*}{$1970-1988$} & \multirow{2}{*}{ 1989-2008 } & \multicolumn{2}{|l|}{ 2009-2010 } \\
\hline & & & Generalistas & Temáticas \\
\hline $\mathrm{N}^{\circ}$ programas & 331 & 723 & 64 & 240 (Inf.)/116(No inf.) \\
\hline $\begin{array}{l}\text { Formatos predominan- } \\
\text { tes }\end{array}$ & $\begin{array}{l}\text { Dibujos animados } \\
\text { Series extranjeras }\end{array}$ & $\begin{array}{l}\text { Series extranjeras } \uparrow \\
\text { Dibujos animados } \downarrow\end{array}$ & \multicolumn{2}{|l|}{$\begin{array}{l}\text { Series extranjeras } \uparrow \\
\text { Dibujos animados } \uparrow\end{array}$} \\
\hline Producción & Extranjera & Extranjera $\uparrow$ & \multicolumn{2}{|l|}{ Extranjera $\uparrow$} \\
\hline Franja horaria & Tarde & $\begin{array}{l}\text { Franja despertador/ } \\
\text { Mañana }\end{array}$ & Franja despertador & $\begin{array}{l}\text { Mañana/Sobremesa } \\
\text { (No inf.) }\end{array}$ \\
\hline Periocidad & Semanal & $\begin{array}{l}\text { De lunes a viernes/ Se- } \\
\text { manal }\end{array}$ & De lunes a viernes & Diaria \\
\hline $\begin{array}{l}\text { Duración media pro- } \\
\text { grama (en ') }\end{array}$ & 38 & 41 & 47 & \\
\hline
\end{tabular}

Fuente: Elaboración propia

1 Tras la prueba piloto llevada a cabo en A Fonsagrada y Soria, el gobierno estableció tres fases para el apagón analógico en España: fase I, hasta el 30 de junio de 2009; fase II, hasta el 31 de diciembre de 2009 y fase III y definitiva con la que se dijo adiós a la era analógica el 2 de abril de 2010 (Sarabia-Andúgar y Sánchez-Martínez, 2012).

2 En este estudio se contabilizaron aquellos programas emitidos de forma independiente. No se tuvieron en cuenta producciones sólo emitidas dentro de programas contenedores. En caso de que empezasen de forma autónoma y luego pasasen a ser contenido de otro o viceversa, únicamente se tuvo en cuenta su emisión por separado. 
A partir de 1989 tuvo lugar el impulso de las televisiones privadas, tanto que en este segundo período Antena 3 y Telecinco aglutinaron más de la mitad de la oferta infantil nacional al emitir más espacios infantiles que los canales públicos. En la segunda etapa (1989-2008), Telecinco se convirtió en la cadena que emitió más programas para niños, sin embargo este interés por este tipo de audiencia se concentró en ocho años, de 1990 a 1998, año en los que dejó de retransmitir diariamente contenidos específicamente infantiles, a excepción de alguna serie juvenil como Al Salir de Clase, 20 tantos o Embrujadas que también desaparecieron de la sobremesa en 2004. Tras Telecinco, La 1 y La 2 siguieron siendo las cadenas que sumaban más programas infantiles en sus correspondientes parrillas.

Cuadro 4: Resumen de la programación infantil de las cadenas generalistas

\begin{tabular}{|c|c|c|c|c|}
\hline & Período & $\mathrm{N}^{\mathrm{o}} \mathrm{y} \%$ programas & Formato & Producción \\
\hline \multirow{3}{*}{ La 1} & 1970-1988 & $262(79 \%)$ & \multirow{14}{*}{$\begin{array}{l}\text { Dibujos animados } \\
\text { Series extranjeras }\end{array}$} & \multirow{14}{*}{ Extranjera } \\
\hline & 1989-2008 & $199(27 \%)$ & & \\
\hline & $2008-2010$ & $1(0,6 \%)$ & & \\
\hline \multirow{3}{*}{ La 2} & 1970-1988 & $107(32 \%)$ & & \\
\hline & 1989-2008 & $188(26 \%)$ & & \\
\hline & 2008-2010 & $14(9 \%)$ & & \\
\hline \multirow{2}{*}{ Antena 3} & 1989-2008 & $152(21 \%)$ & & \\
\hline & $2008-2010$ & $13(8 \%)$ & & \\
\hline \multirow{2}{*}{ Telecinco } & 1989-2008 & 266 (37\%) & & \\
\hline & $2008-2010$ & $12(8 \%)$ & & \\
\hline \multirow{2}{*}{ Cuatro } & 2005-2008 & $27(4 \%)$ & & \\
\hline & 2008-2010 & $16(10 \%)$ & & \\
\hline \multirow{2}{*}{ LaSexta } & 2006-2008 & $12(2 \%)$ & & \\
\hline & $2008-2010$ & $8(5 \%)$ & & \\
\hline
\end{tabular}

Fuente: Elaboración propia

De 2009 a 2010 las cadenas temáticas no infantiles, como Neox, Nova, Telecinco 2, FDF, LaSiete, La10 o SonyTV, emitieron más espacios para niños (116) que las televisiones generalistas (64). Relevante es la parrilla de Neox, que sin ser un canal temático exclusivamente infantil como ClanTV, Disney Channel o Boing, aglutinó sobre el $30 \%$ de la oferta en este período. 
Cuadro 5: Resumen de la programación infantil de cadenas temáticas no infantiles

\begin{tabular}{|c|c|c|c|c|c|c|c|c|}
\hline & \multicolumn{8}{|c|}{ Temáticas No infantiles } \\
\hline & Neox & Nova & FDF & $\begin{array}{l}\text { LaSiete } \\
\text { (mayo2009) }\end{array}$ & $\begin{array}{l}\text { Telecinco } 2 \\
\text { (hasta may.2009) }\end{array}$ & $\begin{array}{l}\text { La10 } \\
\text { (sept.2010) }\end{array}$ & SonyTV & Veo7 \\
\hline \multirow[t]{2}{*}{$\mathrm{N}^{\circ} \mathrm{y} \%$ programas } & $\begin{array}{l}50 \\
(32 \%)\end{array}$ & $\begin{array}{l}8 \\
(5 \%)\end{array}$ & $\begin{array}{l}11 \\
(7 \%)\end{array}$ & $\begin{array}{l}3 \\
(2 \%)\end{array}$ & $\begin{array}{l}20 \\
(13 \%)\end{array}$ & $\begin{array}{l}14 \\
(9 \%)\end{array}$ & $\begin{array}{l}9 \\
(6 \%)\end{array}$ & $\begin{array}{l}1 \\
(0,6 \%)\end{array}$ \\
\hline & \multicolumn{8}{|c|}{ Series extranjeras + Dibujos animados } \\
\hline Producción & \multicolumn{8}{|c|}{ Extranjera } \\
\hline
\end{tabular}

Fuente: Elaboración propia

Más de la mitad de los espacios infantiles emitidos en España tuvo una vida útil ${ }^{3}$ de menos de un año; sin embargo, destaca el porcentaje de programas que ha aparecido más de una vez en antena en un período de más de diez años, que en 2009-2010 supuso el 17\% de la oferta total. De 1975 a 1988 estas producciones más longevas representaban un 12,4\%, menos significativas (11\%) durante la segunda etapa.

Cuadro 6: Algunos de los programas infantiles con más tiempo de vida

\begin{tabular}{|l|l|l|l|}
\hline Al salir de clase & Equipo A, $E l$ & Melrose Place & Salvados por la campana \\
\hline Alf & Gemelas de Sweet Valley & Nanny & Sensación de Vivir \\
\hline Barrio Sésamo & Gran Circo TVE & Padres Forzosos* & Simpson, Los* \\
\hline Bola de Dragón Z & Hércules & Pantera Rosa $^{*}$ & Sonic* $^{*}$ \\
\hline Bonanza & Hora de Bill Cosby, La & Pippi Calzaslargas* $^{*}$ & Spiderman \\
\hline Campeones & Humor amarillo & Power Rangers* & Tribu de los Brady, La \\
\hline Casa de la Pradera, La & Inspector Gadget & Príncipe de BelAir, El* & Trotamundos de Harlem, Los \\
\hline Coche Fantástico, El ${ }^{*}$ & Lois y Clarck & Problemas crecen, Los ${ }^{*}$ & V, Los Visitantes \\
\hline Club Megatrix* & Matrimonio con hijos* & Punky Brewster & Vacaciones en el mar \\
\hline Cosas de Casa* & McGyver & Sabrina, cosas de brujas & Vigilantes de la playa, Los* \\
\hline *Todavía en emisión al finalizar el estudio & \\
\hline
\end{tabular}

Fuente: Elaboración propia

3 Tiempo transcurrido desde la primera emisión hasta la última aparición en antena. 
El tiempo medio en antena ${ }^{4}$ más frecuente en esta tipología de programas fue menor a seis meses. Es común en las tres etapas que más del $80 \%$ de las emisiones tuviese una duración continua de menos de un año. Aun así, es relevante la tendencia a un mayor tiempo en antena en la última etapa, influencia muy posiblemente de las cadenas temáticas. Producciones como Los Simpson, Shin Chan, Padre de Familia, American Dad... continuaban en emisión en el momento de la redacción de este estudio (2015).

Cuadro 7: Algunos de los programas infantiles con más tiempo seguido en antena

\begin{tabular}{|l|l|l|}
\hline Más de 5 años en antena & De 4 a 5 años en antena & De 3 a 4 años en antena \\
\hline Al salir de clase & Aplauso & A media tarde \\
\hline American Dad* & Cesta y puntos & Barrio Sésamo \\
\hline Antena Infantil & Domingo Fiesta & Birlokus Club \\
\hline Chipirifláuticos & Compañeros & Bola de Cristal \\
\hline Cinematrix & Coche Fantástico, El & Cajón Desastre \\
\hline Club Disney & Hormiguero, El* ${ }^{*}$ & Con Vosotros \\
\hline Club Megatrix* & Mundo de la Música, El & Cosas de Casa \\
\hline Conciertazo, El & Padre de Familia* & Gran Circo TVE \\
\hline Gente Joven & Un globo, dos globos... & Guardería, La \\
\hline Hora Warner, La & & Hoy también es Fiesta \\
\hline Lunnis, Los* ${ }^{*}$ & & Libro Gordo de Petete, El \\
\hline Simpson, Los* & & Pista Libre \\
\hline That's English & & Semana, La \\
\hline & & Tocata \\
\hline *Todavía en emisión al finalizar el estudio & ShinChan* \\
\hline
\end{tabular}

Fuente: Elaboración propia

Alude a una emisión continua, sin interrupciones. En caso de ser espacios emitidos por temporadas, como el caso de las series, se re-
fiere a una emisión sin reposiciones y con un ritmo constante. 
Las diversas estrategias de programación se identificaron claramente al analizar las franjas de emisión ${ }^{5}$ de los contenidos infantiles. Así en la primera etapa La $1^{6}$ y La $2^{7}$ reservaron la programación infantil para la sobremesa y en mayor medida para la tarde. A partir de 1989, la mayoría de estos programas pasaron a emitirse en la franja despertador o durante la mañana, cambio impulsado por las televisiones privadas que concentraron la mayor parte de su oferta infantil en la primera parte del día. La cadena pública, para competir con las privadas, también contempló la franja despertador pero las tardes siguieron siendo su horario infantil por excelencia. En esta segunda etapa otra franja horaria que resaltaría sobre las demás fue el access sobremesa, programar contenidos para los niños durante las horas de la comida.

Desde 2009, los canales temáticos no infantiles han propiciado una mayor distribución de la programación infantil durante el día, aunque la franja despertador y la mañana continuaron siendo predominantes. Por su parte la tarde, la franja horaria más afín a los niños, sí que perdió toda su relevancia de años anteriores y ya se emitían más contenidos a la hora de comer que de 18.00 a 20.00. En esta última etapa resultó interesante el incremento de espacios para un público infantil y juvenil en prime time, una estrategia potenciada principalmente por Antena 3 y Neox al emitir series nacionales de corte juvenil (El Internado, Física o Química, Los Protegidos) y otros programas para toda la familia pero con protagonismo infantil (¿Sabes más que un niño de primaria?, Cinematrix) en horario de máxima audiencia.

Las diferentes estrategias de programación de las cadenas influyeron de forma directa en los cambios que los programas infantiles sufrieron en las parrillas televisivas. Como consecuencia, se analizó la evolución que la programación infantil sufrió en las parrillas de las cadenas generalistas:

- La 1: La tarde se convirtió en la franja horaria más recurrente para la emisión de programación infantil en La 1. Durante más de 15 años la primera cadena pública retransmitió contenidos para los más pequeños todos los días de la semana de 18.00 a 20.00. Posiblemente por la competencia de las televisiones privadas, en 1992 incluyó dibujos animados a primera hora de la mañana, una estrategia que duró poco más de tres años durante los días laborables pero que mantuvo los fines de semana.

5 Las franjas horarias utilizadas por Kantar Media son: franja mañana (7.00-14.00), sobremesa (14.00-17.00), tarde (17.00-20.30), prime time (20.30-24.00), late night (00.00-2.30) y madrugada (2.30-7.00).

$6 \quad$ La 1 no cuenta con las franjas horarias 'despertador' y matinal hasta 1986. En 1975 sus emisiones comenzaban a las 13.45. En 1984 se amplió 15 minutos el período de emisión para finalmente iniciar a las 7.15 en enero de 1986. Los fines de semana la carta de ajuste se colocaba más temprano que durante la semana, sobre las 11.45, que pasó a ser sobre las 10.40 los sábados y sobre las 9.30 los domingos a partir de 1981 .

7 La 2 no cuenta con las franjas horarias 'despertador' y matinal hasta 1989. Hasta prácticamente esa fecha, el segundo canal público emitía de tarde, comenzando sus emisiones sobre las 20.00-19.00 en un primer momento para luego empezar sobre las 18.00. En 1978, empezó a ofrecer contenidos durante la sobremesa de los fines de semana. En 1985 La 2 inició emisiones a última hora de la mañana pero también solo los sábados y domingos. 
Desde 1996 la directiva de la cadena pública retiró de la parrilla diaria los contenidos infantiles para concentrarlos en las mañanas de sábados y domingos (siempre que los eventos deportivos lo permitiesen) y en La 2.

Cuadro 8: Franjas horarias en las que La 1 emitió programación infantil

\begin{tabular}{|c|c|c|c|c|c|c|c|}
\hline \multirow{2}{*}{$\begin{array}{c}\text { FRANIA } \\
\text { HORARIA }\end{array}$} & \multicolumn{7}{|c|}{ PERIODO - De Lunes a Viernes } \\
\hline & \multirow[t]{8}{*}{$1975-1986$} & \multirow[t]{3}{*}{$1987-1989$} & \multirow[t]{5}{*}{ 1990-1991 } & 1992-1993 & \multirow[t]{5}{*}{1994} & 1995 & \multirow[t]{8}{*}{$1996-2010$} \\
\hline Despertador & & & & & & & \\
\hline Matinal & & & & & & & \\
\hline Access Sobremesa & & & & & & & \\
\hline Sobremesa & & & & & & & \\
\hline Tarde & & & & & & & \\
\hline Access PrimeTime & & & & & & & \\
\hline PrimeTime & & & & & & & \\
\hline
\end{tabular}

\begin{tabular}{|c|c|c|c|c|c|c|c|c|c|c|c|c|}
\hline \multirow{3}{*}{$\begin{array}{c}\text { FRANJA } \\
\text { HORARIA }\end{array}$} & \multicolumn{12}{|c|}{ PERIODO - Fin de semana } \\
\hline & 1975 & 1977 & 1979 & 1980 & 1982 & 1984 & \multicolumn{2}{|c|}{$1986-1990$} & 1991 & 1993 & 1995 & 2008-2010 \\
\hline & \multirow[t]{8}{*}{$S \quad D$} & \multirow[t]{2}{*}{$\begin{array}{ll} & D\end{array}$} & 5 & S D & S D & $S \quad D$ & 5 & D & 50 & S D & $S D$ & $\mathbf{S}$ \\
\hline Despertador & & & & & & & & & & & & \\
\hline Matinal & & & & & & & & & & & & \\
\hline Access Sobremesa & & & & & & & & & & & & \\
\hline Sobremesa & & & & & & & & & & & & \\
\hline Tarde & & & & & & & & & & & & \\
\hline Access PrimeTime & & & & & & & & & & & & \\
\hline PrimeTime & & & & & & & & & & & & \\
\hline
\end{tabular}

Fuente: Elaboración propia

La 2: Hasta 1990, la parrilla de La 2 prescindió en varias ocasiones de la programación infantil en los días laborables. Durante los primeros años de la década de los 90, estos contenidos tampoco gozaron de gran estabilidad al fluctuar entre las franjas despertador, access sobremesa y tarde. A partir de 1996, año en el que se retiró de la oferta diaria de La 1 todos los programas infantiles, La 2 se convirtió en la cadena pública a servicio de los niños, emitiendo para ellos a primera hora de la mañana, a mediodía, a la tarde y antes del horario de máxima audiencia. A partir de 2010, los contenidos para los niños cesaban a las 18.00. Los fines de semana de La 2 no se caracterizaron por ofrecer una oferta estable. Si bien durante la semana fue sustituyendo a La 1 como contenedor infantil, los sábados y domingos siempre se dedicaron a complementar la programación de la primera cadena. 
Cuadro 9: Franjas horarias en las que La 2 emitió programación infantil

\begin{tabular}{|c|c|c|c|c|c|c|c|c|c|c|c|c|c|c|}
\hline \multirow{2}{*}{$\begin{array}{c}\text { FRANJA } \\
\text { HORARIA }\end{array}$} & \multicolumn{14}{|c|}{ PERIODO - De Lunes a Viernes } \\
\hline & \multirow[t]{8}{*}{ 1975-1981 } & \multirow[t]{7}{*}{ 1982-1987 } & \multirow[t]{8}{*}{ 1988-1989 } & 1990 & 1991 & \multirow[t]{8}{*}{1992} & \multirow[t]{8}{*}{1993} & 1994 & \multirow[t]{3}{*}{1995} & 1996 & $1997-2005$ & $2006-2007$ & $2008-2009$ & 2010 \\
\hline Despertador & & & & & & & & & & & & & & \\
\hline Matinal & & & & & & & & & & & & & & \\
\hline Access Sobremesa & & & & & & & & & & & & & & \\
\hline Sobremesa & & & & & & & & & & & & & & \\
\hline Tarde & & & & & & & & & & & & & & \\
\hline Access PrimeTime & & & & & & & & & & & & & & \\
\hline PrimeTime & & & & & & & & & & & & & & \\
\hline
\end{tabular}

\begin{tabular}{|c|c|c|c|c|c|c|c|c|c|c|c|c|c|}
\hline \multirow{3}{*}{$\begin{array}{c}\text { FRANJA } \\
\text { HORARIA }\end{array}$} & \multicolumn{13}{|c|}{ PERIODO - Fin de semana } \\
\hline & \multirow{2}{*}{$\begin{array}{l}1975 \\
5 \quad D\end{array}$} & \begin{tabular}{|l}
$1977-1980$ \\
\end{tabular} & 1981 & $1982-1984$ & 1984-1985 & 1986 & 1988 & 1991 & $1993-1996$ & 1997 & 1999 & 2001 & \begin{tabular}{|l|l|}
$2002-2010$ \\
\end{tabular} \\
\hline & & D & $S \quad D$ & $\mathrm{~s}$ & $\mathrm{~s}$ & 50 & $S \quad D$ & $S \quad D$ & $\mathrm{~s}$ & $\begin{array}{ll}5 & 0\end{array}$ & & $S \quad D$ & D \\
\hline Despertador & & & & & & & & & & & & & \\
\hline Matinal & & & & & & & & & & & & & \\
\hline Access Sobremesa & & & & & & & & & & & & & \\
\hline Sobremesa & & & & & & & & & & & & & \\
\hline Tarde & & & & & & & & & & & & & \\
\hline Access PrimeTime & & & & & & & & & & & & & \\
\hline PrimeTime & & & & & & & & & & & & & \\
\hline
\end{tabular}

Fuente: Elaboración propia

Antena 3: fue la primera cadena en programar contenido para niños en la franja despertador, una estrategia que mantuvo durante casi 20 años los siete días de la semana. El access sobremesa también fue una franja horaria por la que esta cadena sigue apostando en la actualidad (desde 1993) al optar por la emisión de comedias familiares (Cosas de Casa, Salvados por La Campana, Príncipe de Bel Air, Sabrina, Padres Forzosos, Cosas de Marcianos...) y de Los Simpson en las horas de la comida. En los primeros años de vida de la cadena la programación infantil se emitía además de tarde, una franja del día que Antena 3 decidió dedicar a otros públicos desde 1996. Desde 2006 Antena 3 redujo su programación infantil diaria reservando solamente el mediodía. Hasta la fecha límite de estudio en este trabajo (diciembre 2010), este canal compensaba esa limitación semanal retransmitiendo durante toda la mañana del fin de semana su programa contenedor más conocido, Club Megatrix. Como particularidad, Antena 3 dedicó ocasionalmente (1993; 2004 y 2008) la franja de máxima audiencia de los sábados a la emisión de películas destinadas a un público infantil. 
Cuadro 10: Franjas horarias en las que Antena 3 emitió programación infantil

\begin{tabular}{|c|c|c|c|c|c|c|c|c|}
\hline \multirow{2}{*}{$\begin{array}{l}\text { FRANJA } \\
\text { HORARIA }\end{array}$} & \multicolumn{7}{|c|}{ PERIODO - De Lunes a Viernes } & \multirow{3}{*}{2010} \\
\hline & 1990 & 1991-1992 & 1993-1996 & $1997-2000$ & 2001-2003 & \multirow[t]{3}{*}{ 2004-2005 } & 2006-2009 & \\
\hline \multirow{2}{*}{\multicolumn{7}{|c|}{ Despertador }} & & \\
\hline \multirow{2}{*}{\multicolumn{9}{|c|}{ 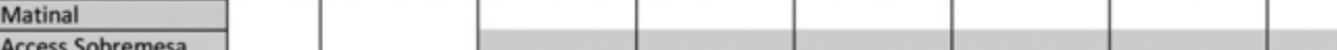 }} \\
\hline \multicolumn{2}{|l|}{ Access Sobremesa } & & & & & & & \\
\hline \multicolumn{9}{|l|}{ Sobremesa } \\
\hline \multicolumn{9}{|l|}{ Tarde } \\
\hline \multicolumn{9}{|l|}{ Access PrimeTime } \\
\hline PrimeTime & & & & & & & & \\
\hline
\end{tabular}

\begin{tabular}{|c|c|c|c|c|c|c|c|c|c|c|c|c|c|c|c|c|c|}
\hline \multirow{3}{*}{$\begin{array}{l}\text { FRANJA } \\
\text { HORARIA }\end{array}$} & \multicolumn{17}{|c|}{ PERIODO - Fin de semana } \\
\hline & 1991 & \multicolumn{2}{|c|}{1993} & \multicolumn{2}{|c|}{1995} & \multicolumn{2}{|c|}{ 1997-1999 } & \multicolumn{2}{|c|}{$2001-2002$} & \multicolumn{2}{|c|}{2004} & \multicolumn{2}{|c|}{2006} & \multicolumn{2}{|c|}{2008} & \multicolumn{2}{|c|}{2010} \\
\hline & S D & s & $\mathrm{D}$ & $s$ & & $\mathrm{~s}$ & D & 5 & D & & D & & & & D & & D \\
\hline Despertador & & & & & & & & & & & & & & & & & \\
\hline Matinal & & & & & & & & & & & & & & & & & \\
\hline Access Sobremesa & & & & & & & & & & & & & & & & & \\
\hline Sobremesa & & & & & & & & & & & & & & & & & \\
\hline Tarde & & & & & & & & & & & & & & & & & \\
\hline Access PrimeTime & & & & & & & & & & & & & & & & & \\
\hline PrimeTime & & & & & & & & & & & & & & & & & \\
\hline
\end{tabular}

Fuente: Elaboración propia

Telecinco: El compromiso de Telecinco con la programación infantil fue intenso en los primeros años de la década de los noventa, pero también fugaz al desatender prácticamente a este público desde 1998 durante los días laborables (hasta 2003 emitió Al salir de clase en horario de sobremesa). Así, empezó televisando dibujos animados durante la tarde (1990 a 1993) a la que se unió la franja despertador que se convirtió en la más duradera al permanecer inalterable desde 1991 a 1997. A finales de la década de los 90 la cadena decidió cambiar su estrategia de programación y trasladar los contenidos infantiles y juveniles de la primera hora de la mañana a la sobremesa para propiciar su completa desaparición de la parrilla en 2003. Igual que Antena 3, Telecinco aprovechó las mañanas del sábado y domingo para completar su oferta infantil que en los primeros años también se prolongaba hasta la tarde. 
Cuadro 11: Franjas horarias en las que Telecinco emitió programación infantil

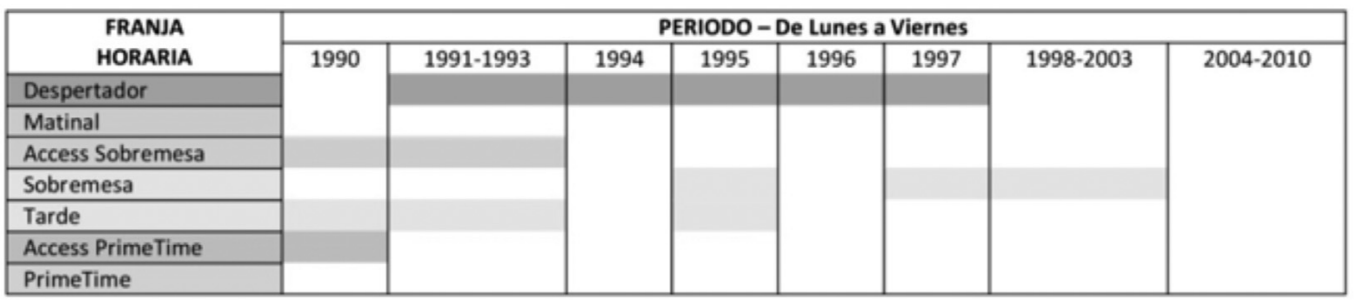

\begin{tabular}{|c|c|c|c|c|c|c|c|c|c|c|c|c|c|}
\hline \multirow{3}{*}{$\begin{array}{l}\text { FRANJA } \\
\text { HORARIA }\end{array}$} & \multicolumn{13}{|c|}{ PERIODO - Fin de semana } \\
\hline & 1991 & \multicolumn{2}{|c|}{1993} & \multicolumn{2}{|c|}{1995} & 1997 & \multicolumn{2}{|c|}{$1999-2004$} & \multicolumn{2}{|c|}{2006} & \multicolumn{2}{|c|}{2008} & 2010 \\
\hline & S D & $\mathrm{s}$ & D & $\mathrm{s}$ & D & $\mathrm{s}$ & $\mathrm{s}$ & D & & & & & $\mathrm{s}$ \\
\hline Despertador & & & & & & & & & & & & & \\
\hline Matinal & & & & & & & & & & & & & \\
\hline Access Sobremesa & & & & & & & & & & & & & \\
\hline Sobremesa & & & & & & & & & & & & & \\
\hline Tarde & & & & & & & & & & & & & \\
\hline Access PrimeTime & & & & & & & & & & & & & \\
\hline PrimeTime & & & & & & & & & & & & & \\
\hline
\end{tabular}

Fuente: Elaboración propia

Cuatro: Igual que el resto de cadenas privadas, Cuatro optó desde sus inicios (hasta 2010) por atraer al espectador infantil a primera hora de la mañana. Como todo canal en sus inicios, probó emitir contenidos para niños en otras franjas horarias, como el momento de la comida y la sobremesa, que pronto caerían de la parrilla. No obstante, una franja que dedicó a un público más familiar fue el inicio del prime time al programar de lunes a jueves a las 21.30 El Hormiguero (que ya se había estrenado en 2006 en horario de sobremesa), con contenidos dirigidos especialmente a los niños. Se mantuvo en emisión hasta su marcha en 2011 a Antena 3. 
Cuadro 12: Franjas horarias en las que Cuatro emitió programación infantil

\begin{tabular}{|c|c|c|c|c|c|c|}
\hline \multirow{2}{*}{$\begin{array}{l}\text { FRANJA } \\
\text { HORARIA }\end{array}$} & \multicolumn{6}{|c|}{ PERIODO - De Lunes a Viernes } \\
\hline & 2005 & 2006 & 2007 & 2008 & 2009 & 2010 \\
\hline \multicolumn{7}{|l|}{ Despertador } \\
\hline \multicolumn{7}{|l|}{ Matinal } \\
\hline \multicolumn{7}{|l|}{ Access Sobremesa } \\
\hline \multicolumn{7}{|l|}{ Sobremesa } \\
\hline \multicolumn{7}{|l|}{ Tarde } \\
\hline \multicolumn{7}{|l|}{ Access PrimeTime } \\
\hline PrimeTime & & & & & & \\
\hline
\end{tabular}

\begin{tabular}{|c|c|c|c|c|c|c|}
\hline \multirow{3}{*}{$\begin{array}{l}\text { FRANIA } \\
\text { HORARIA }\end{array}$} & \multicolumn{6}{|c|}{ PERIODO - Fin de Semana } \\
\hline & \multicolumn{2}{|c|}{2006} & \multicolumn{2}{|c|}{2008} & \multicolumn{2}{|c|}{2010} \\
\hline & & D & $\mathrm{s}$ & D & $s$ & D \\
\hline \multicolumn{7}{|l|}{ Despertador } \\
\hline \multicolumn{7}{|l|}{ Matinal } \\
\hline \multicolumn{7}{|l|}{ Access Sobremesa } \\
\hline \multicolumn{7}{|l|}{ Sobremesa } \\
\hline \multicolumn{7}{|l|}{ Tarde } \\
\hline \multicolumn{7}{|l|}{ Access PrimeTime } \\
\hline PrimeTime & & & & & & \\
\hline
\end{tabular}

Fuente: Elaboración propia

La Sexta: La cadena generalista privada más reciente eligió la sobremesa para emitir contenidos concebidos para un público infantil y/o juvenil (Padre de Familia, Futurama...). A diferencia de los otros canales que recurrían al fin de semana para reforzar su oferta infantil, La Sexta dedicaba sábados y domingos a otros perfiles de espectadores, en especial a aquellos interesados en los eventos deportivos. 
Cuadro 13: Franjas horarias en las que La Sexta emitió programación infantil

\begin{tabular}{|c|c|c|c|c|}
\hline \multirow{2}{*}{$\begin{array}{l}\text { FRANJA } \\
\text { HORARIA }\end{array}$} & \multicolumn{4}{|c|}{ PERIODO - De Lunes a Viernes } \\
\hline & \multirow[t]{8}{*}{2006} & \multirow[t]{4}{*}{2007} & 2008 & \multirow[t]{4}{*}{ 2009-2010 } \\
\hline Despertador & & & & \\
\hline Matinal & & & & \\
\hline Access Sobremesa & & & & \\
\hline Sobremesa & & & & \\
\hline Tarde & & & & \\
\hline Access PrimeTime & & & & \\
\hline PrimeTime & & & & \\
\hline
\end{tabular}

\begin{tabular}{|c|c|c|c|c|c|c|}
\hline \multirow{3}{*}{$\begin{array}{l}\text { FRANJA } \\
\text { HORARIA }\end{array}$} & \multicolumn{6}{|c|}{ PERIODO - Fin de Semana } \\
\hline & \multicolumn{2}{|c|}{2006} & \multicolumn{2}{|c|}{2008} & \multicolumn{2}{|c|}{2010} \\
\hline & $\mathrm{s}$ & D & 5 & $\mathrm{D}$ & 5 & D \\
\hline \multicolumn{7}{|l|}{ Despertador } \\
\hline \multicolumn{7}{|l|}{ Matinal } \\
\hline \multicolumn{7}{|l|}{ Access Sobremesa } \\
\hline \multicolumn{7}{|l|}{ Sobremesa } \\
\hline \multicolumn{7}{|l|}{ Tarde } \\
\hline \multicolumn{7}{|l|}{ Access PrimeTime } \\
\hline PrimeTime & & & & & & \\
\hline
\end{tabular}

Fuente: Elaboración propia

Directamente relacionada con la evolución de las franjas horarias de contenido infantil, está la tendencia descendente en la media de horas diarias dedicadas a esta audiencia. A excepción de La 2, (que aunque en 2010 ya se empieza a apreciar su decrecimiento en el número de minutos), todas las televisiones fueron disminuyendo el tiempo dedicado al público infantil. Entre 1990 y 1995 la programación infantil vivió su momento de esplendor al disponer de espacios tanto de mañana como de tarde en todas las parrillas nacionales. La 2 y Antena 3 fueron las cadenas generalistas nacionales que más tiempo dedicaron a los niños (hasta 8 horas al día); por su parte Telecinco, aunque en sus primeros pasos contempló a este perfil de audiencia (destinando hasta 5 horas/día), perdió su interés en un período corto de tiempo. 
Cuadro 14: Media de horas diarias dedicadas a la programación infantil

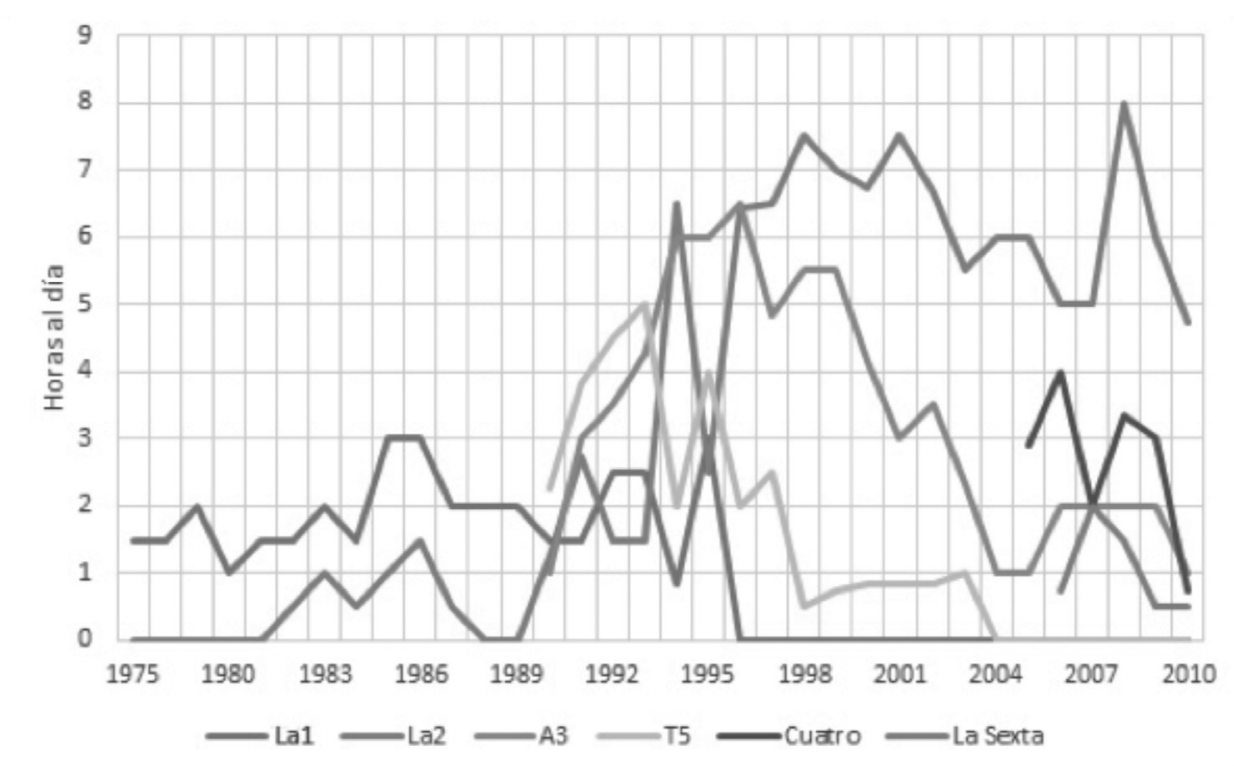

Fuente: Elaboración propia

Un nexo común a las tres etapas analizadas fue la duración de los espacios. Lo más habitual era que cada emisión durase 30 minutos, a excepción de los programas contenedores u otros de entretenimiento que al menos se extendían durante una hora. Con el transcurso de los años se observó una tendencia a dilatar las emisiones: si en la primera etapa, la media de duración de un programa era de 38 minutos, entre 1989 y 2008 fue de 41 minutos hasta alcanzar los 47 minutos en el período 2009-2010.

También es interesante cómo ha evolucionado la frecuencia con la que se emiten los espacios infantiles. Durante el monopolio de la televisión pública, la periodicidad de los programas infantiles era semanal, es decir, cada día de la semana se emitía una oferta diferente. Con la aparición de las cadenas privadas, se impuso una emisión diaria de lunes a viernes, aunque la semanal seguía siendo recurrente. Durante la segunda etapa, programar contenidos de forma específica para el fin de semana o para períodos estivales o Navidad. En la última época la emisión en días laborables se mantuvo como la frecuencia de programación preferente, pero cada vez era más común la retransmisión del mismo producto los siete días de la semana, de lunes a domingo.

La tipología de programación infantil en España se ha reducido a la elección de dibujos animados y de series extranjeras, que representan más del 70\% de todas las emisiones en las tres etapas. En los primeros años, la animación superaba en 
volumen a la ficción, situación que cambió en la década de los 90 y en los primeros años del nuevo milenio al perder los dibujos animados representatividad frente a la ficción foránea. A partir de 2009, la animación volvió a ganar presencia e igualó en estadísticas a las series.

Además de dibujos animados y series de ficción, en cada época tuvo relevancia, en menor medida, otro tipo de producciones. Durante el monopolio, además de entretener, la cadena pública también buscaba formar e informar, de ahí que durante esta etapa fuese frecuente la emisión de programas educativos, de idiomas, de divulgación científica, de variedades (magia y circo) o informativos juveniles. Los programas contenedores, más bien sus precursores, también fueron un formato recurrente que se afianzó en la segunda etapa de la mano de las televisiones generalistas privadas. Telecinco, más proclive a la renovación de sus contenedores, y Antena 3 más constante (Club Megatrix es uno de los programas más longevos de la oferta infantil) dieron impulso a este formato que con el paso del tiempo y la aparición de las cadenas temáticas perdió fuerza y su representatividad se redujo progresivamente. En el tercer período, motivado por las cadenas temáticas, la telenovela infantil se convirtió en el género más usado tras las series de ficción extranjeras y los dibujos animados, emitidos en su mayoría por Nova, La Siete y SonyTV.

La procedencia de la mayoría de los contenidos infantiles es extranjera con una tendencia al alza. Si hasta 1988, la programación infantil foránea suponía un 74,6\%, en la segunda etapa llegó al 79\% para situarse en un 86\% en el tercer período de análisis. La televisión pública emitía más producción nacional que las privadas, aunque Antena 3 y en mayor medida Telecinco también mostraron en un primer momento su compromiso por el producto audiovisual hecho en España.

$\mathrm{Al}$ analizar la programación infantil, siempre se hace alusión a la repetición de contenidos en las parrillas. En este caso se comprobó que la media de reposición de este tipo de programación ha ido en aumento conforme pasaron los años. De 1975 a 1988 la media de repeticiones de un programa fue de 1,86, en la segunda etapa ya cada producto se reponía dos veces, mientras que a partir de 2009 la tasa de repetición alcanzó el 2,41. Este incremento del número de veces que se retransmite un mismo contenido puede implicar una menor tendencia a la renovación de contenidos, una característica que muchos expertos (Cebrián Herreros, 2003; Vázquez Barrio, 2011) suelen atribuir a la programación infantil. 
Cuadro 15: Programas infantiles que fueron repuestos en antena al menos cinco veces

\begin{tabular}{|l|l|}
\hline Alf & McGyver \\
\hline Casa de la Pradera, La & Nanny \\
\hline Coche Fantástico, El & Pantera Rosa, La \\
\hline Cosas de marcianos & Príncipe de Bel-Air, El \\
\hline Embrujadas & Problemas Crecen, Los \\
\hline Equipo A, El & Punky Brewster \\
\hline Gemelas de Sweet Valley, Las & Salvados por la Campana \\
\hline Hércules & Sensación de Vivir \\
\hline Lois y Clarck & Vigilantes de la Playa, Los \\
\hline Matrimonio con Hijos & Una chica explosiva \\
\hline
\end{tabular}

Fuente: Elaboración propia

En esta línea, también se intentó reflejar el nivel de estabilidad que tenía la programación infantil en las parrillas de televisión. Se comprobó que el número de programas que sufre algún tipo de modificación -cambios de hora, de día, de frecuencia de emisión, hasta de cadena- es significativo (cuatro de cada diez) y se incrementa en cada etapa. Si en la década de los 80 un 43\% de los programas emitidos sufrió algún cambio, en 2010 la cifra ascendió al 60\%.

La mayor parte de las variables empleadas para el estudio de la programación infantil de las cadenas generalistas carecen de relevancia científica para analizar las temáticas infantiles en abierto, ya que sus parrillas están concebidas para emitir exclusivamente contenido infantil. De 2009 a 2010 Clan TV, Disney Channel y Boing (que salió al aire en septiembre de 2010) retransmitieron un total de 240 programas, en su mayoría dibujos animados y series de ficción extranjeras. La tasa de renovación de contenidos entre 2009 y 2010 fue casi de un $60 \%{ }^{8}$ por lo que durante estos dos años mantuvieron en antena aproximadamente el $43 \%$ de sus producciones. En relación con la exclusividad de sus contenidos, un 16\% del total de la programación emitida durante este período de análisis ya había sido ofertada previamente por cadenas generalistas, en su mayoría por aquellas pertenecientes al mismo grupo de comunicación que las temáticas infantiles.

8 En los cálculos de este porcentaje no se incluyó a Boing dado su nacimiento en septiembre de 2010. 
Cuadro 16: Resumen de la programación infantil de cadenas temáticas infantiles

\begin{tabular}{|l|l|l|l|}
\hline \multirow{2}{*}{} & Temáticas infantiles & \multicolumn{2}{|l|}{} \\
\cline { 2 - 4 } & ClanTV & Disney Channel & Boing (sept.2010) \\
\hline No programas & 104 & 106 & 32 \\
\hline Formato & Dibujos animados + Series extranjeras \\
\hline Producción & Extranjera & \\
\hline
\end{tabular}

Fuente: Elaboración propia

\section{Conclusiones}

Tras revisar 40 años de emisiones regulares de la televisión española se puede concluir que la programación infantil ha tenido, en líneas generales, una presencia decreciente en las parrillas de las cadenas generalistas. Tras una 'época dorada' en la década de los 90 en la que canales como Antena 3 o Telecinco emitieron diariamente contenidos para los niños tanto por la mañana como por la tarde, una oferta para un público más generalista (telenovelas, concursos, películas, magazines...) ha ido sustituyendo a los programas infantiles que se convirtieron, a partir del año 2000, en contenido relegado a las mañanas del fin de semana y posteriormente -con la aparición y consolidación de la TDT-a las parrillas de los canales temáticos. Consecuentemente, en la última etapa de análisis (2009-2010) las cadenas temáticas no infantiles en abierto ya programaban más contenido infantil que los canales generalistas.

De forma más concreta:

- $\quad$ La 1 dejó de emitir diariamente programas para niños desde 1996, que pasaron a retransmitirse por la segunda cadena.

- Dos años después Telecinco siguió la misma estrategia al retirar de su programación diaria toda oferta infantil, a excepción de las series con temática juvenil en horario de sobremesa (Al Salir de Clase o 20 tantos), en antena hasta 2004, cuando fueron reemplazadas por programas de actualidad.

- $\quad$ Antena 3 tardaría unos años más, hasta 2006, para retirar su oferta infantil de la franja despertador y dejar únicamente en antena Los Simpson al mediodía (una serie que en 2015 sigue manteniendo su horario de emisión).

- $\quad$ Las últimas cadenas generalistas en salir al aire, Cuatro y La Sexta, nunca se caracterizaron por tener una oferta infantil estable y definida.

- La franja horaria de tarde, tradicionalmente asociada a un público infantil, fue la primera que los programadores decidieron desligar de los niños para ofrecer otro tipo de contenidos dirigido a otros perfiles de audiencia, como amas de casa y/o personas jubiladas. 
- L L L programación infantil también fue desapareciendo de la franja despertador y se mantuvo hasta la última etapa de análisis (2009-2010) aquellas franjas horarias tradicionalmente menos afines al público infantil, tal como la sobremesa o el access prime time.

- A tenor de los resultados obtenidos se puede concluir que el nivel de atención brindado a la programación infantil por las cadenas generalistas ha ido decreciendo por su falta de interés en este tipo de contenidos y que se puede comprobar en los siguientes datos:

- $\quad$ Casi un 20\% de los programas analizados se volvió a reponer en antena más de una vez en un período de 10 años; producciones como Bola de Dragón Z, El Coche Fantástico, El Equipo A, Bonanza, Los Vigilantes de la Playa han sufrido múltiples reposiciones lo que se traduce como un síntoma de la poca renovación a la que se ven sometidos los contenidos ofertados a los niños.

- $\quad$ No se puede afirmar que la programación infantil haya gozado de estabilidad en las parrillas de las cadenas generalistas porque se detectó que seis de cada diez programas analizados sufrieron alguna alteración (cambios de hora, de día, de cadena, interrupciones por programaciones especiales...) durante su tiempo medio en antena que además no solía ser superior a seis meses, lo que implicaba al mismo tiempo una oferta con poca vocación de continuidad.

- Los principales productos programados para una audiencia infantil son las series y los dibujos animados, mayoritariamente de producción extranjera.

Relacionado con lo anterior, las series de ficción de las cadenas generalistas no suelen dirigirse de forma específica a los niños, no obstante se inclinan por un protagonismo coral con personajes de diversas edades y condición social con el objetivo de atraer al mayor porcentaje de audiencia (Vázquez Barrio, 2011). Por ejemplo, Física o Química o El Internado tienen como protagonistas a un grupo de jóvenes con tramas que transcurren en el entorno escolar para buscar la atención de los niños. Que productoras y cadenas optasen por esta temática permite vislumbrar el interés por captar la atención del espectador joven, aunque algunas de estas producciones no sean recomendables para menores de 16 años.

Del mismo modo que ocurre con la ficción, dibujos animados como Padre de Familia o American Dad tampoco son aptos para un público infantil. Esta restricción no fue una traba para que las cadenas programasen estas producciones en las franjas horarias en las que más niños están viendo la televisión (de 17.00 a 20.00 y de 20.00 a 22.00).

Muchas de las producciones que los canales tratan como programación para niños (sobre todo series tanto extranjeras como nacionales y dibujos animados) no son precisamente aptas para un espectador menor de 12 años. Las series animadas para un público adulto (Los Simpson, Padre de Familia, American Dad) suscitan gran controversia, ya que a pesar de ser concebidas para mayores, su formato de animación atrae a gran cantidad de niños y su calificación puede que no 
sea la más adecuada (por ejemplo, en el caso de Los Simpson, recomendado para todos los públicos). De todos modos, para profundizar en esta cuestión sería necesario un estudio concreto que analizase los contenidos de cada programa, lo que se aleja de los alcances de esta investigación.

\section{Referencias bibliográficas}

Asociación para la investigación de medios de comunicación (2014): Resumen general EGM de octubre 2014 a mayo 2015. Disponible en: http://www.aimc.es/spip.php?action=acceder_document\&arg=2877\&cle=4aa7ca0bfee3e9a848f0eleb c984975a5ab14fe5\&file=pdf\%2Fresumegm215.pdf [Consultado el 11/10/2015].

Barlovento Comunicación (2011): Análisis televisivo 2010. Disponible en: http:/ /www.periodistadigital.com/documentos/2011/01/04/ANALISIS\%20TELEVISIVO\%202010.pdf [Consultado el 05/10/2015].

Bringué, X. (2001): “Publicidad infantil y estrategia persuasiva: un análisis de contenido", Zer Revista de Estudios de Comunicación, n. 10, pp. 107-130.

Cebrián Herreros, M. (2003): “Contenidos infantiles en televisión. Nueva técnica analítica global”, Zer Revista de Estudios de Comunicación, n. 15, pp. 51-65.

Código de Autorregulación sobre Contenidos Televisivos e Infancia. Disponible en: http://tvinfancia.es/tvinfancia/ sites/default/files/codigo.pdf [Consultado el 23/09/2015].

Kantar Media. (2012): Anuario 2012 de audiencias de televisión. Recuperado de CD-ROM prestado en Biblioteca de la Facultade de Ciencias Sociais e da Comunicación, Universidade de Vigo.

McNeal, J. U. (1992): Kids as Customers: A Handbook of Marketing to Children. Nueva York: Lexington Books.

Melgarejo, I. y Rodríguez, M. M. (2013): "El lado amable de la televisión: los canales infantiles politemáticos educativos y su uso didáctico en la familia y en la escuela”, XI Encuentro del Consejo escolar de la Región de Murcia: crecer entre pantallas, los medios de comunicación y la educación, pp. 133-151.

Ortiz, M. A.; Ruiz, J. A. y Díaz, E. (2013): “Las televisiones y la investigación en infancia y televisión”, Comunicar, 20 (40), pp. 137-144. DOI: http://dx.doi.org/10.3916/C40-2013-03-04

Pérez Alonso-Geta, P. M. (dir.), y Sánchez Peris, F. (coord.) (2010): Estudio sobre el comportamiento de los consumidores de juegos y juguetes (8-12 años): valores, perfiles y preferencias. Disponible en: http://www.observatoriodeljuego.es/db_archivos/11_4.pdf [Consultado el 12/09/2015].

Ruiz, M. (s. f.): "Desarrollo del niño como consumidor", Psicología online. Disponible en: http://www.psicologiaonline.com/psicologia_de_las_organizaciones/el-nino-consumidor.html [Consultado el 22/09/2015].

Roedder, D. (1999): “Consumer socialization of children: a retrospective look at twentyfive years of research”, Journal of Consumer Research, 26 (3), pp. 183- 220. 
Salgado Carrión, J. A. (2006): La presencia de la televisión en los hábitos de ocio de los niños. Madrid: Fundación Autor, D.L. Sarabia-Andúgar, I. y Sánchez-Martínez, J. (2012): “Adaptación de los hogares a la televisión digital terrestre en su primera fase de implantación en España”, Revista Latina de Comunicación Social, n. 67, pp. 68-98. DOI: 10.4185/RLCS-067-948-068098.

Sierra Bravo, R. (2001): Técnicas de investigación social: ejercicios y problemas. Madrid: Paraninfo.

Tur Viñes, V. y Ramos Soler, I. (2008): Marketing y niños. Madrid: ESIC Editorial.

Vázquez Barrio, T. (2011): ¿Qué ven los niños en la televisión? Madrid: Universitas. 